\title{
Multiple Scattering Approximation of Anti-plane Elastic Waves in Infinite and Half-Space Domains with Distributed Inclusions
}

\author{
Kwannate Tharmmapornphilas and Sohichi Hirose \\ Department of Mechanical and Environmental Informatics, Tokyo Institute of \\ Technology \\ 2-12-1 O-okayama, Meguro-ku, Tokyo, 152-8552, Japan \\ Email: shirose@cv.titech.ac.jp
}

\begin{abstract}
This paper presents an approximation for multiple scattering of elastic waves in the frequency domain by cylindrical inclusions. The problem is reduced to a $2 \mathrm{D}$ model by assuming circular inclusions. These inclusions are distributed at randomly selected points in $2 \mathrm{D}$ isotropic solids. Infinite and half-space matrices are considered. The inclusions are subjected to an anti-plane (SH) incident wave. The proposed approximation is based on the assumption that the multiple scattering displacement is the summation of the effects of all the possible wave propagation paths. If a wave hits one inclusion, the wave is scattered and part of it scatters to the calculation point. The wave also scatters to the other inclusions and thus repeating the process. This process is repeated a lot of times but the scattered wave becomes smaller as the path length increases and thus becomes negligible up to a certain order. Each of these scattered waves is approximated using the displacements calculated using the boundary element method with farfield approximation for a single scatterer. Using the proposed approximation, the computational time and the memory requirement are considerably reduced as compared to the conventional boundary element method. Numerical results for two aligned inclusions, thirty randomly selected and hundred randomly placed inclusions in both infinite and half-space matrices are shown to verify the accuracy of the proposed approximation.
\end{abstract}


Key words: boundary element method, elastic wave, far field, multiple scattering

\section{Introduction}

Among nondestructive evaluation techniques, ultrasonic testing is widely used in many industries and is recognized as the most useful tool in quality control of materials and structures [1]. High precision of ultrasonic testing is required to detect very small defects in materials. However, such high frequency testing faces difficulty in detecting defects in materials with large grains. In such materials, flaw echoes are usually weaker than the noise echoes caused by the scattering at the grain boundary. Thus for such cases, flaw echoes are difficult to distinguish from noise echoes and therefore defects cannot be evaluated properly.

Noises generally found in the typical ultrasonic testing system can be defined and categorized into electric circuit noises, ringing noises, digital noises of digital systems, and structural noises caused by the grain boundaries of materials [2]. Structural noises are considered the most difficult noises to analyze and manage, while the other noises can be reduced significantly by using digital noise reduction filters. Therefore, the scattering characteristics of elastic waves in materials with scatterers must be studied in order to understand the nature of the structure noises. A main approach to clarify and understand the nature of noises is to carry out numerical calculations of elastic wave scattering in materials. Due to the interaction between scatterers, the multiple scattering model should be considered. The numerical analysis of multiple scattering of anti-plane waves with long wave lengths was performed by Biwa et al. [3]. Saitoh et al. applied fast multipole boundary element method for scattering problem in composites [4]. Cai et. al. introduce formulations of multiple scattering of anti-plane wave and a method called scatterer polymerization[5-6]. Unfortunately, current numerical models for multiple scattering require very high computational time and larger computer memory leading to difficulty in calculation. Therefore, several approximation methods have been proposed and developed to solve such problems.

Some researches have been dedicated in developing approximation methods for 
scattering problems [7-10]. The Born approximation, applied to elastodynamics of wave scattering by flaws and inclusions presented by Gubernatis et al. [7] and Rose et al. [8], was shown to be effective for weak scattering models. The extended quasi-static approximation, proposed by Gubernatis et al. [9], combines assumptions of the Born approximation and the low frequency static limit. Darmon et al. modified the extended quasi-static approximation by adding a new assumption, the so-called doubly distorted wave [10].

In this paper, an approximation method based on the use of boundary element method is proposed for multiple scattering problems. The multiple scattered displacement is expressed as the summation of the displacements of waves from all possible wave scattering propagation paths. The scattered displacement of each wave scattering propagation path is the product of the single scattered displacements along the propagation path. Each single scattered displacement is calculated using conventional BEM with farfield approximation. Numerical models of infinite and half-space matrices containing randomly distributed cylindrical inclusions are analyzed by both the multiple scattering approximation and the conventional BEM for comparison of the results.

\section{Boundary element method for scattering from one inclusion}

In this paper, the boundary element method is used to calculate the wave scattering by one inclusion and is used as the reliable method for the reference results. The scattering displacement by one inclusion in an infinite matrix subjected to a unit anti-plane wave is used as a multiplier in the multiple scattering approximation. The scattering process, in which a unit anti-plane wave is incident to an inclusion as illustrated in Fig. 1, is called the single scattering process. The scattered displacement obtained from this process is called the single scattered displacement.

Consider an anti-plane wave motion in a homogeneous elastic solid with a homogenous elastic inclusion as shown in Fig. 1. The equation of anti-plane harmonic motion is 


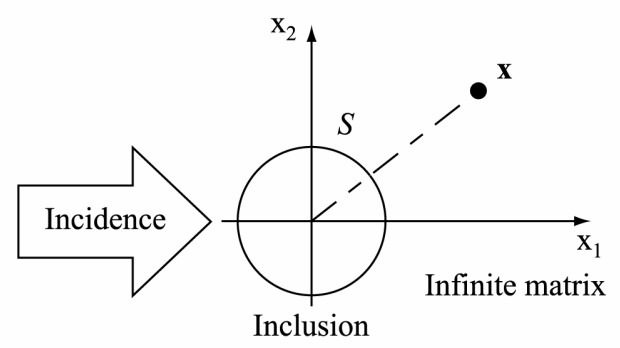

Fig. 1 Single scattering model

$\mu \nabla^{2} u_{3}(\mathbf{x}, \omega)+\rho \omega^{2} u_{3}(\mathbf{x}, \omega)=0, \mathbf{x} \in D$

where $\rho$ is the density, $\mu$ is the shear modulus, $\omega$ is frequency and $u_{3}$ is the anti-plane displacement.

For 2D problems, the boundary integral equation for anti-plane displacement in a homogeneous isotropic elastic matrix is [11]

$$
\begin{aligned}
\frac{1}{2}\left(u_{3}^{\mathrm{M}}(\mathbf{x})-u_{3}^{\mathrm{in}}(\mathbf{x})\right)= & \int_{S}\left[U_{33}^{\mathrm{M}}(\mathbf{x}, \mathbf{y}) t_{3}^{\mathrm{M}}(\mathbf{y})-T_{33}^{\mathrm{M}}(\mathbf{x}, \mathbf{y}) u_{3}^{\mathrm{M}}(\mathbf{y})\right] d S(\mathbf{y}) \\
& -\int_{S}\left[U_{33}^{\mathrm{M}}(\mathbf{x}, \mathbf{y}) t_{3}^{\mathrm{in}}(\mathbf{y})-T_{33}^{\mathrm{M}}(\mathbf{x}, \mathbf{y}) u_{3}^{\mathrm{in}}(\mathbf{y})\right] d S(\mathbf{y}) .
\end{aligned}
$$

For displacements on the boundary of an elastic inclusion, the boundary integral equation can be written as

$$
\frac{1}{2} u_{3}^{\mathrm{I}}(\mathbf{x})=\int_{S}\left[U_{33}^{\mathrm{I}}(\mathbf{x}, \mathbf{y}) t_{3}^{\mathrm{I}}(\mathbf{y})-T_{33}^{\mathrm{I}}(\mathbf{x}, \mathbf{y}) u_{3}^{\mathrm{I}}(\mathbf{y})\right] d S(\mathbf{y})
$$

where $u_{3}$ and $t_{3}$ are the displacement and the traction, respectively. The superscripts $\mathrm{M}$, I, and 'in' refer to the matrix, the inclusion, and the incident wave, respectively. $S$ denotes the boundary of the inclusion and the matrix. $\mathbf{x}$ and $\mathbf{y}$ are arbitrary points on the boundary. The fundamental solutions $U_{33}$ and $T_{33}$ can be written as

$U_{33}(\mathbf{x}, \mathbf{y})=\frac{i}{4 \mu} H_{0}^{(1)}\left(k_{\mathrm{T}} r\right)$ 
$T_{33}(\mathbf{x}, \mathbf{y})=-\frac{i}{4} k_{\mathrm{T}} \frac{\partial r}{\partial n_{\mathrm{y}}} H_{1}^{(1)}\left(k_{\mathrm{T}} r\right)$

where $H_{0}{ }^{(1)}$ denotes the zero order Hankel function of the first kind and $r=|\mathbf{x}-\mathbf{y}| . k_{\mathrm{T}}, \mu$, and $n_{y}$ are the shear wave number, the shear modulus, and the unit normal vector in the outward direction at $\mathbf{y}$, respectively. Assuming perfect bond between the matrix and the inclusion, the boundary conditions are given by

$u_{3}^{\mathrm{I}}(\mathbf{x})=u_{3}^{\mathrm{M}}(\mathbf{x})$

$t_{3}^{\mathrm{I}}(\mathbf{x})=-t_{3}^{\mathrm{M}}(\mathbf{x})$

when $\mathbf{x}$ is a point on the boundary. $u_{3}{ }^{\mathrm{M}}$ and $t_{3}^{\mathrm{M}}$ can be obtained numerically by substituting Eqs. (4), (5), (6), and (7) into Eqs. (2) and (3) and solving the discretized boundary integral equations.

After solving for $u_{3}{ }^{\mathrm{M}}$ and $t_{3}{ }^{\mathrm{M}}$, the scattering displacement at an arbitrary point $\mathbf{x}$ in the matrix can be calculated from

$u_{3}^{\mathrm{sc}}(\mathbf{x})=\int_{S}\left[U_{33}^{\mathrm{M}}(\mathbf{x}, \mathbf{y}) t_{3}^{\mathrm{M}}(\mathbf{y})-T_{33}^{\mathrm{M}}(\mathbf{x}, \mathbf{y}) u_{3}^{\mathrm{M}}(\mathbf{y})\right] d S(\mathbf{y})$

Using the farfield approximation where $|\mathbf{x}| \gg|\mathbf{y}|$, the Hankel function can be approximated by

$H_{n}^{(1)}(k r) \approx \sqrt{\frac{2}{\pi k r}} \exp \left[i\left\{k r-(2 n+1) \frac{\pi}{4}\right\}\right]$.

Using Eq. (9), Eq. (8) can be reduced to [12]

$u_{3}^{\text {sc:ar }}(\mathbf{x})=\Omega(\hat{\mathbf{x}}) D\left(k_{\mathrm{T}}^{\mathrm{M}}|\mathbf{x}|\right)$

where 


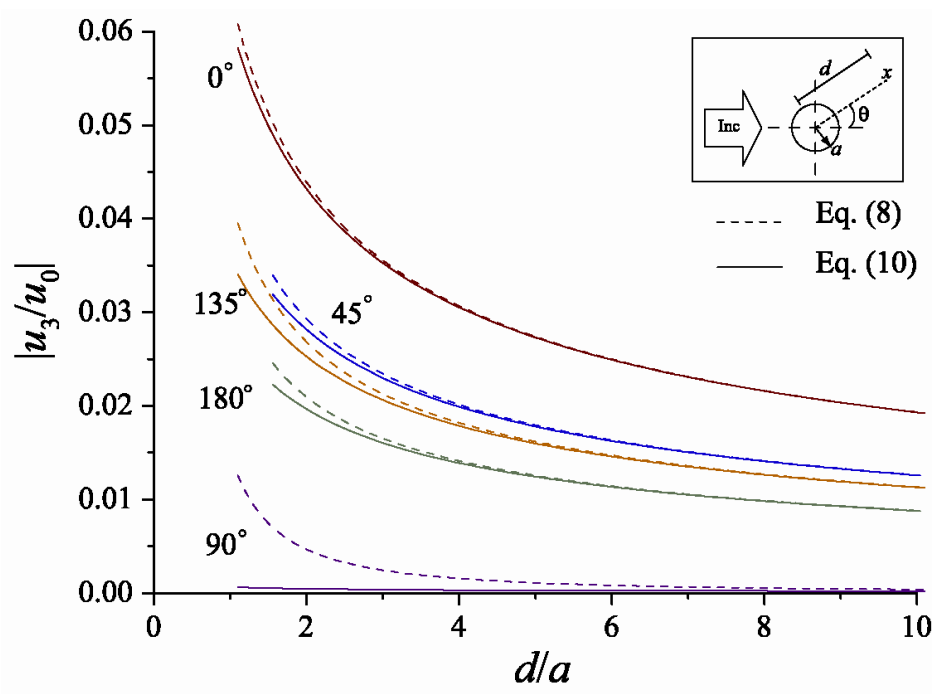

Fig. 2 Comparison of the scattered displacements calculated by Eqs. (8) and (10) with $a k=1$.

$\Omega(\hat{\mathbf{x}})=\int_{S} e^{-i k_{\mathrm{T}}^{\mathrm{M}} \hat{\mathbf{x}} \cdot \mathbf{y}} t_{3}^{\mathrm{M}}(\mathbf{y}) d S(\mathbf{y})-i k_{\mathrm{T}}^{\mathrm{M}} \hat{x}_{j} \int_{S} n_{j}(\mathbf{y}) e^{-i k_{\mathrm{T}}^{\mathrm{M}} \hat{\mathbf{x}} \cdot \mathbf{y}} u_{3}^{\mathrm{M}}(\mathbf{y}) d S(\mathbf{y})$

$D\left(k_{\mathrm{T}}^{\mathrm{M}}|\mathbf{x}|\right)=\frac{\exp \left[i\left(k_{\mathrm{T}}^{\mathrm{M}}|\mathbf{x}|+\pi / 4\right)\right]}{\sqrt{8 \pi k_{\mathrm{T}}^{\mathrm{M}}|\mathbf{x}|}}$

where $\hat{\mathbf{x}}=\mathbf{x} /|\mathbf{x}|$.

To verify the accuracy of far field approximation, the comparison between the scattered displacements calculated using the far field approximation of Eq. (10) and those without approximation (Eq. (8)) are shown in Fig. 2. Both scattered displacements are shown for different scattering angles, $\theta$, and normalized distances, d/a. The displacements calculated with the approximation show good agreement to those without approximation when $d / a$ is greater than 3 . If the scattering angle is $90^{\circ}$, the error is large when $d / a$ is less than 3. However, the values of the scattered displacements at this angle are small. Therefore, this error has a small effect on the displacement of the scattering 


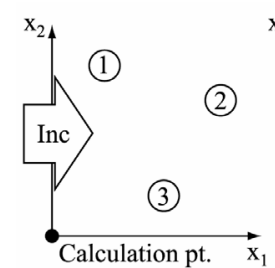

(a) Model

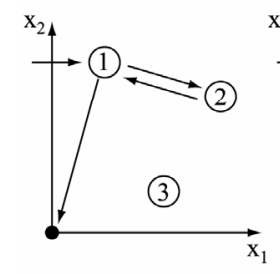

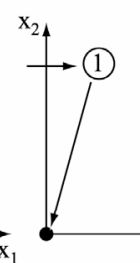

(b) First order
(2)

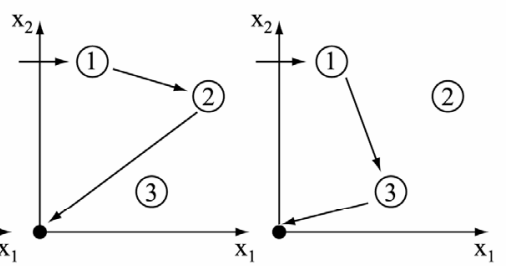

(c) Second order

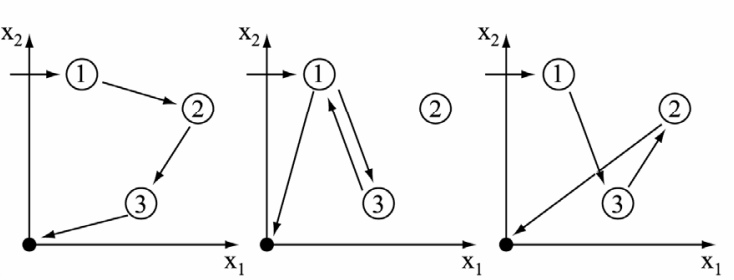

(d) Third order

Fig. 3 Possible wave scattering propagation paths of the third order approximation if an incident wave first hits inclusion number 1 for three inclusions problem

model. Note that, the error of approximation increases with decreasing $a k$. However, the scattered displacement is small such that the error can be assumed negligible.

\section{Multiple scattering approximation}

In this study the multiple scattering approximation is proposed for both scattering problems in the infinite domain and the half-space domain. The multiple scattering approximation is based on the assumption that the multiple scattering displacement is the summation of the displacements of all possible wave scattering propagation paths. One wave scattering propagation path refers to the possible connecting paths between inclusions that a wave travels before going to the calculation point. In this paper, the order of wave scattering propagation path is classified according to the number of inclusions that scatters the wave before it returns to the calculation point. Higher orders can be neglected because the scattered waves become smaller after each scattering process. Figure 3 shows all possible wave scattering propagation paths in the third order approximation in which an incident wave is first incident to the inclusion number 1 in 


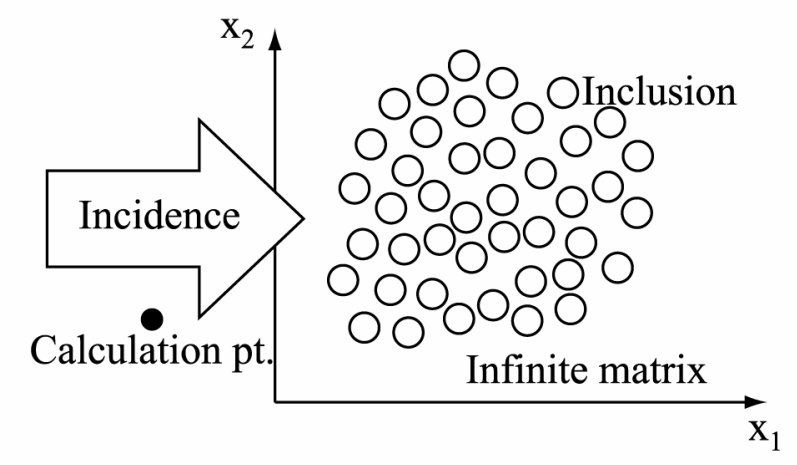

Fig. 4 Numerical model of inclusions randomly distributed in an infinite domain

three inclusions problem.

\subsection{Multiple scattering of waves in the infinite domain}

The model for the multiple scattering approximation of circular inclusions in infinite matrix is illustrated in Fig. 4. A plane wave is incident onto a number of cylindrical inclusions, which are distributed at randomly selected point in the infinite matrix. The scattered displacement at an arbitrary point in the matrix is calculated.

Considering up to the $K^{\text {th }}$ order, the multiple scattering approximation displacement is a summation of displacement of $1^{\text {st }}$ to $K^{\text {th }}$ order wave scattering propagation path at an arbitrary point x. For two-dimensional inclusion problems in an infinite domain, the multiple scattering approximation equation can be expressed as

$U_{3}^{K^{\text {th }} \text { order }}(\mathbf{x})=\sum_{N=1}^{K} u_{3}^{N^{\text {th }} \text { order }}(\mathbf{x})$

where the scattered displacement of all possible $N^{\text {th }}$ order wave scattering propagation paths is

$u_{3}^{N^{\text {th }} \text { order }}(\mathbf{x})=\sum_{\text {all possible }}\left[\left(\prod_{j=1}^{N} R_{P_{j}}\right) u_{3}^{\text {inc }}\left(\mathbf{x}_{P_{1}}\right)\right]$ 
where $u_{3}^{\text {inc }}$ is the incident wave. For each wave scattering propagation path, the inclusions are numbered according to the wave propagation process as $P_{1}, P_{2}, \ldots, P_{N}$. Inclusion $P_{1}$ is the first inclusion that the wave hits. Inclusion $P_{N}$ is the last inclusion that scatters the wave. $R_{P_{j}}$ is the single scattering coefficient obtained by calculating the displacement at the center point of the inclusion $P_{j+1}$. This displacement is due to the scattering by the inclusion $P_{j}$ of a unit wave incident from the center point of inclusion $P_{j-1}$. Using the functions of $D$ and $\Omega$ defined in Eqs. (11) and (12), $R_{P_{j}}$ can be written in an explicit form as

$R_{P_{j}}=D\left(k_{\mathrm{T}}\left|\mathbf{x}_{P_{j}+1}-\mathbf{x}_{P_{j}}\right|\right) \Omega\left(\theta_{P_{j}}\right)$

where $\mathbf{x}_{P_{j}}$ is the center point for the inclusion $P_{j}$ and $\theta_{P_{j}}$ is the angle between the segments $\mathbf{x}_{P_{j}}-\mathbf{x}_{P_{j-1}}$ and $\mathbf{x}_{P_{j+1}}-\mathbf{x}_{P_{j}}$.

According to farfield approximation, the scattered displacement in Eq. (10) is a function of direction and distance. To reduce the computational time, $\Omega$, which is the function of $\theta_{P_{i}}$, is calculated and then stored in the memory. In each scattering process, $\Omega$ is used in order to obtain the single scattered displacement.

\subsection{Multiple scattering of waves in the half-space domain}

The numerical model of a half-space problem is shown in Fig. 5. A plane wave is incident from the left of the inclusions randomly distributed in the half-space, which has a free boundary at $\mathrm{x}_{1}=0$ (see Fig. 5(a)). By mirroring the inclusions and the incident wave as shown in Fig. 5(b), the free boundary can be removed. Then, this problem can be analyzed using the multiple scattering approximation for an infinite domain. The multiple scattering approximation in Eq. (13) is modified and written for the half-space 


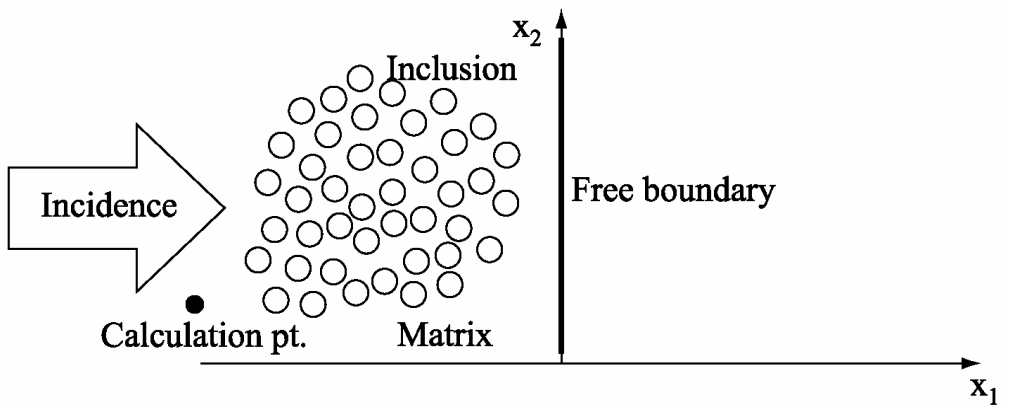

(a)

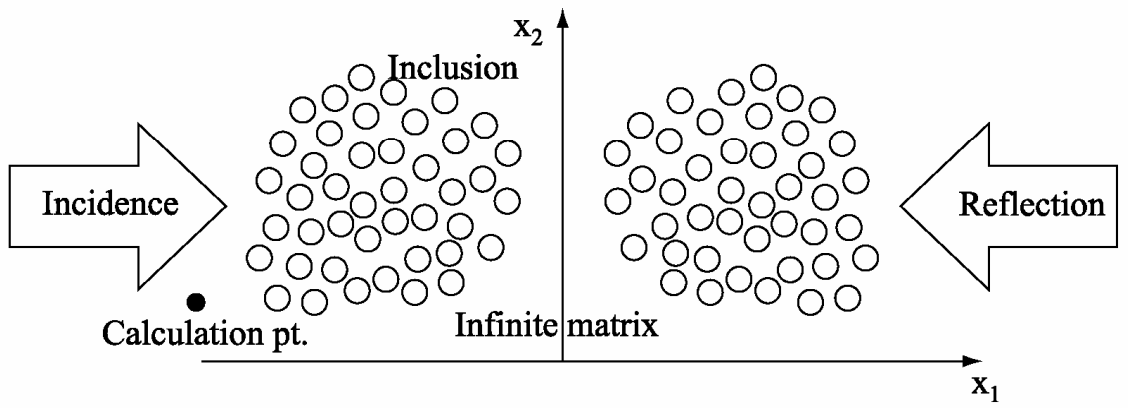

(b)

Fig. 5 Numerical model for half-space problem

problem as

$U_{3}^{K^{\text {th }} \text { order }}(\mathbf{x})=\sum_{N=1}^{K}\left[\sum_{\text {all possible }}\left\{\left(\prod_{j=1}^{N} R_{P_{j}}\right) u_{3}^{\text {inc }}\left(\mathbf{x}_{P_{1}}\right)\right\}+\sum_{\text {all possible }}\left\{\left(\prod_{j=1}^{N} R_{P_{j}}\right) u_{3}^{\text {ref }}\left(\mathbf{x}_{P_{1}}\right)\right\}\right]+u_{3}^{\text {ref }}(\mathbf{x})(16)$

where the superscript 'ref' stands for the reflected wave from the right.

\section{Numerical examples}

Numerical models of two-aligned inclusions, thirty randomly placed inclusions and hundred randomly placed inclusions are shown for infinite and half-space domains. Let $c_{\mathrm{I}}$ and $c_{\mathrm{M}}$ be the shear wave velocity of the inclusion and the matrix, respectively. The 


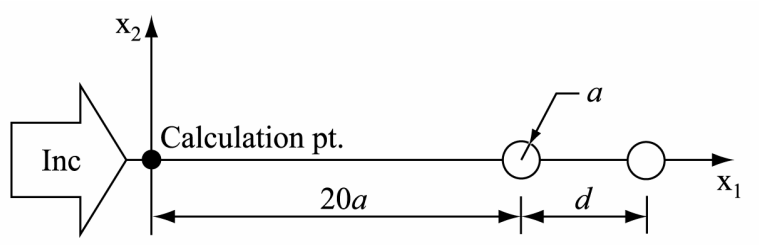

Fig. 6 Two-inclusion model in an infinite domain

velocity ratio of the inclusion and the matrix $\left(c_{\mathrm{I}} / c_{\mathrm{M}}\right)$ is chosen as 1.05 because the simple model for grain noise problem is considered. Note that increasing the velocity ratio leads to an increase in the error of the results. The displacement results of each model computed using the proposed multiple scattering approximation are compared with those of the conventional BEM. In the BEM analysis, 40 constants elements are used for the discretization of the boundary of each inclusion.

\subsection{Two-inclusion model}

Two inclusions with radius $a$ are aligned parallel to the incident wave direction in an infinite matrix as shown in Fig. 6. The distance between the two inclusions, $d$, is taken as $3 a, 5 a$, and $10 a$. The numerical results of the multiple scattering approximation, considered up to the $4^{\text {th }}$ order approximations, are compared to the conventional BEM results and are shown in Fig. 7. Figures 7(a), (b), and (c) are the results for the cases of $d=3 a, 5 a$, and $10 a$, respectively. The $\mathrm{X}$-axis is the wave number normalized by inclusion radius and the Y-axis is the absolute value of the normalized scattered displacement normalized by incident wave at the calculation point. In all figures, the red, blue, purple green and black color lines represent results of the $1^{\text {st }}, 2^{\text {nd }}, 3^{\text {rd }}, 4^{\text {th }}$ multiple scattering approximations and the conventional BEM, respectively. Comparing to the BEM result, error resulted from the multiple scattering approximation increases in the case of small distance, $d$, and high wave number. This is a result of higher scattering interaction between the two inclusions. The periodicity of wave depends on the relation between the distance, $d$, and the wave length. The periodicity of the envelope of wave is dependent on the relation between the inclusion size and wave length. 


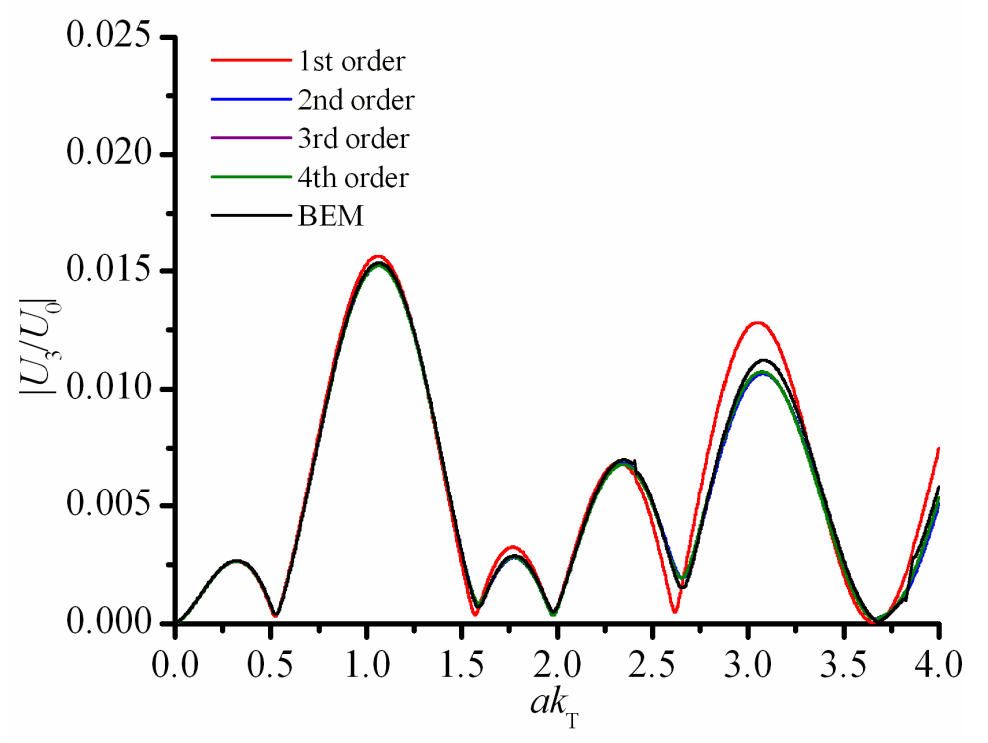

(a) $d=3 a$

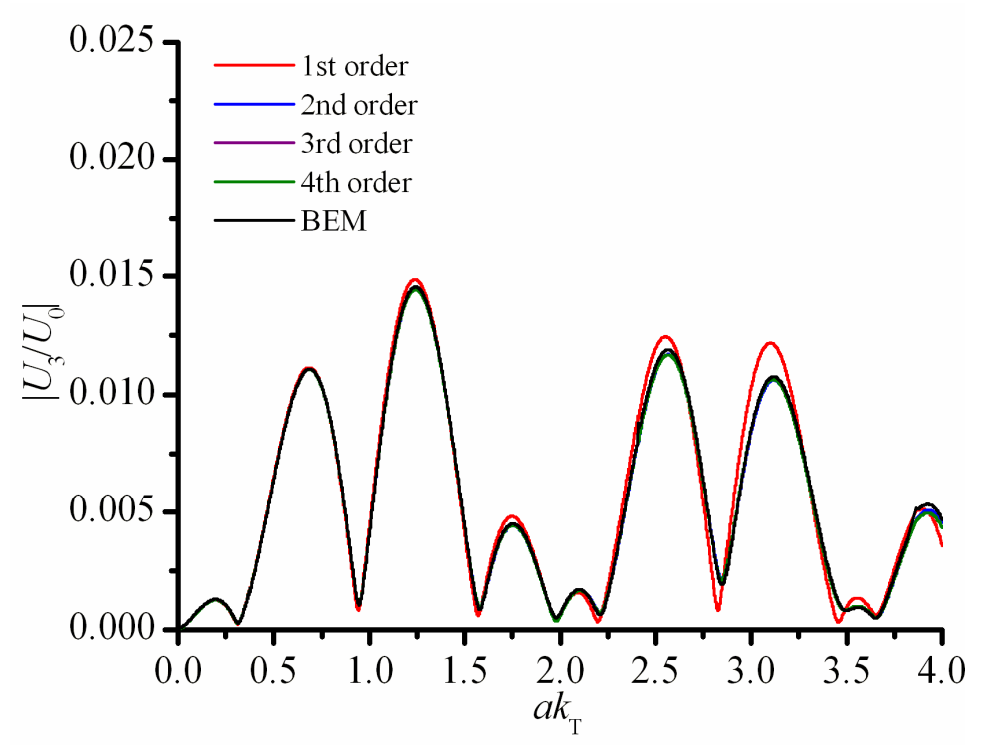

(b) $d=5 a$

Fig. 7 Numerical results of scattered displacement for an anti-plane wave in infinite domain for two inclusions aligned parallel to an incident wave propagation direction, $c_{\mathrm{I}} / c_{\mathrm{M}}=1.05$ 


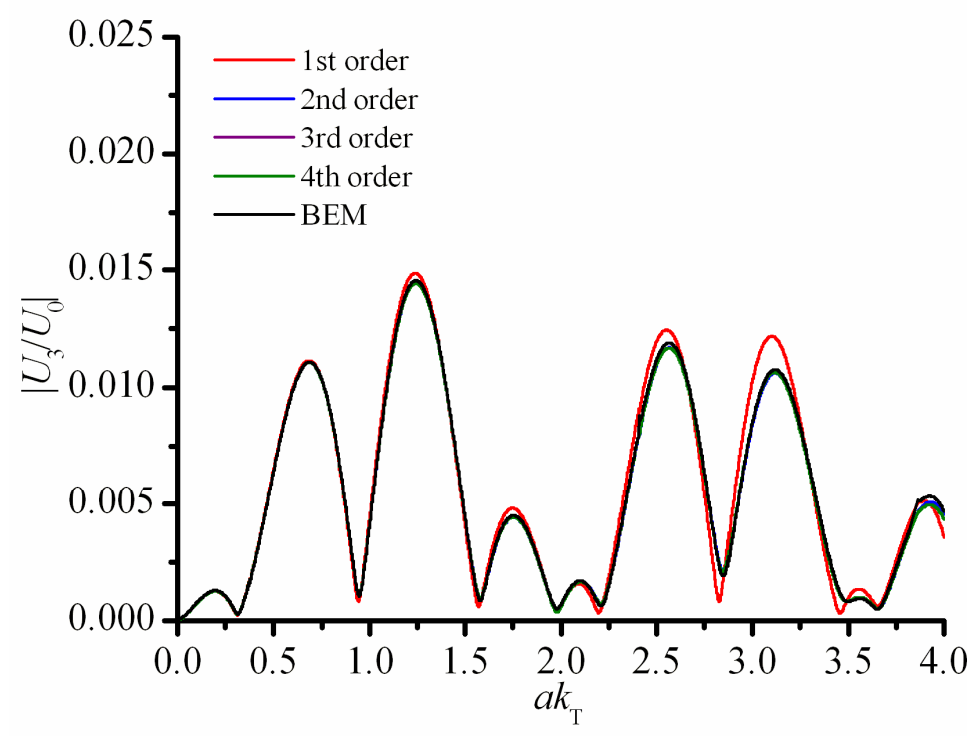

(c) $d=10 a$

Fig. 7(continue) Numerical results of scattered displacement for an anti-plane wave in infinite domain for two inclusions aligned parallel to an incident wave propagation direction, $c_{\mathrm{I}} / c_{\mathrm{M}}=1.05$

Considering the BEM results as the reference results, Figure 8 shows the relative error of the results for each order of approximation. The $\mathrm{X}$-axis is the normalized wave number and the Y-axis is the absolute value of the difference between the scattered displacement results at the calculation point from the multiple scattering approximation and from the BEM results. It is obvious that the results of the $2^{\text {nd }}, 3^{\text {rd }}$ and $4^{\text {th }}$ order approximations show better results than the $1^{\text {st }}$ order approximation in the range of considered wave number. The results show the importance and necessity of higher order multiple scattering approximation. The errors of the $2^{\text {nd }}, 3^{\text {rd }}$, and $4^{\text {th }}$ orders are almost the same. The errors decease with increasing distance $d$. 


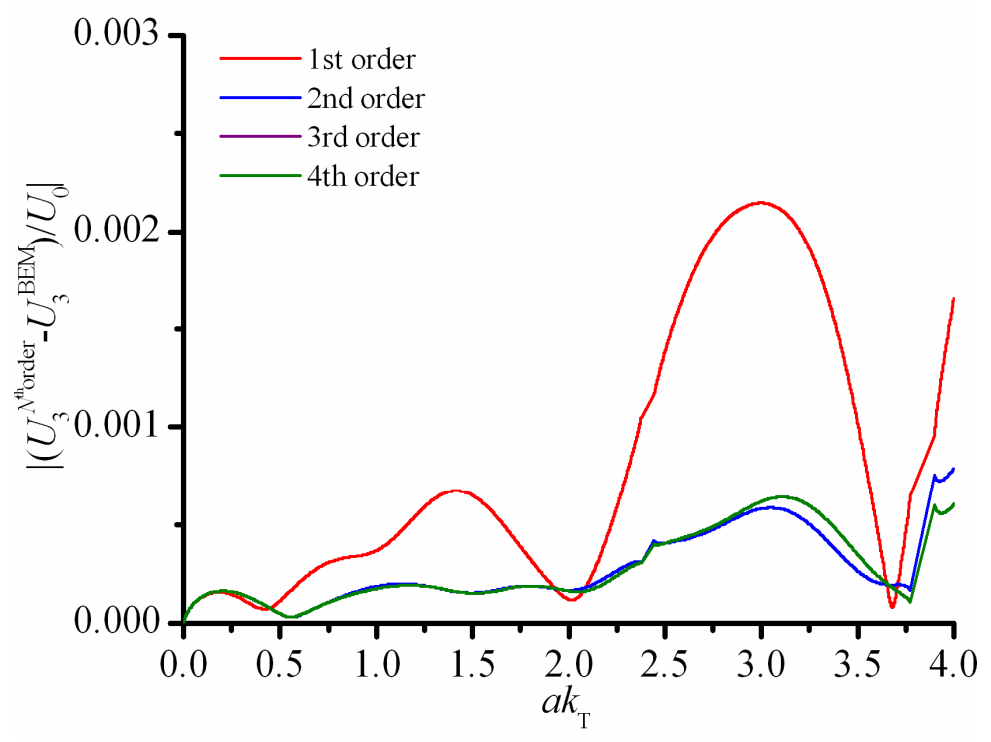

(a) $d=3 a$

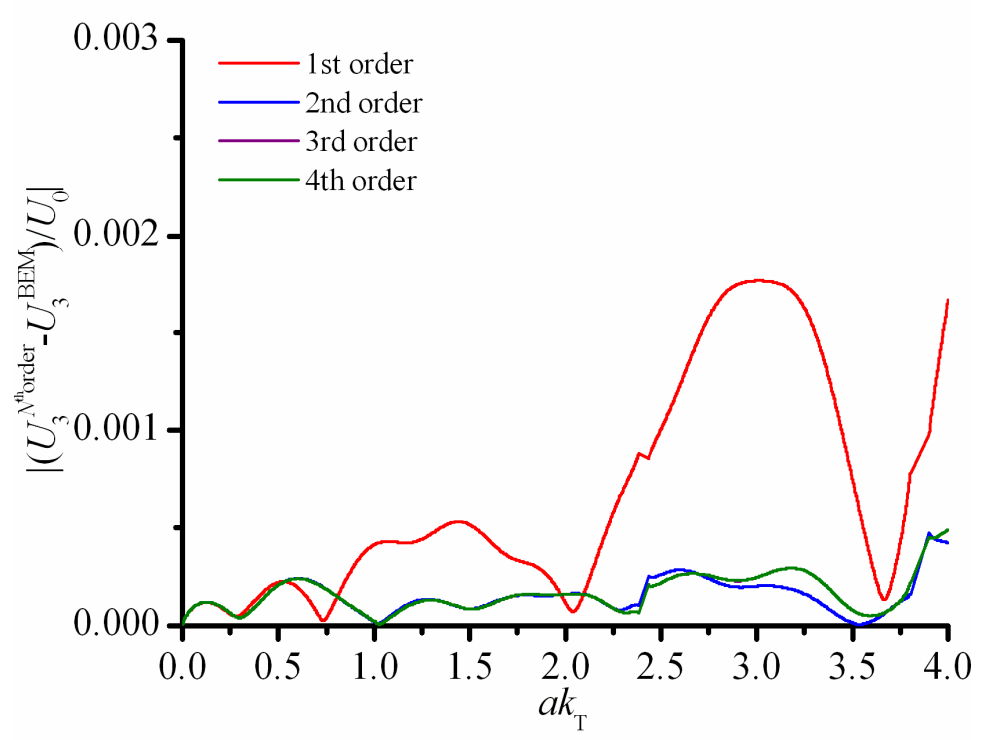

(b) $d=5 a$

Fig. 8 Relative error of the result of scattered displacement for an anti-plane wave in infinite domain for two inclusions aligned parallel to an incident wave propagation direction, $c_{\mathrm{I}} / c_{\mathrm{M}}=1.05$ 


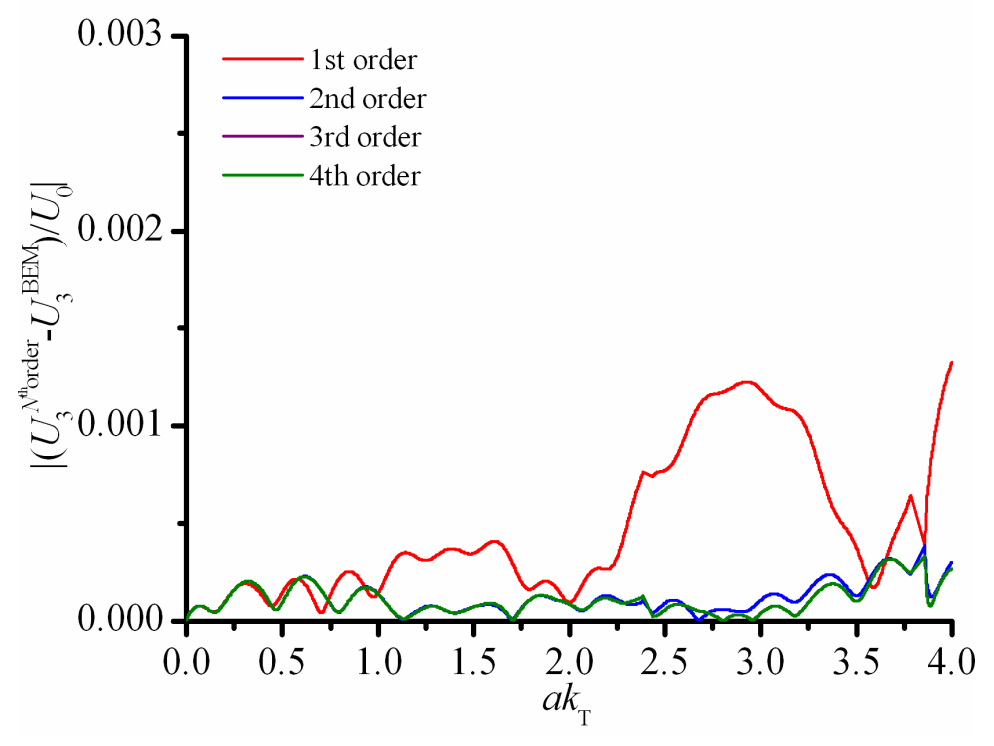

(c) $d=10 a$

Fig. 8 (continue) Relative error of the result of scattered displacement for an anti-plane wave in infinite domain for two inclusions aligned parallel to an incident wave propagation direction, $c_{\mathrm{I}} / c_{\mathrm{M}}=1.05$

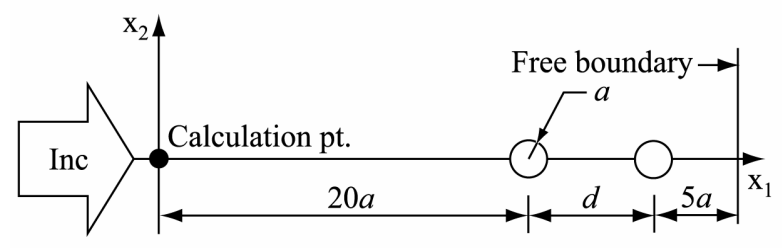

Fig. 9 Two inclusions model in a half-space domain

Figure 9 shows a model of two inclusions in a half space domain. The free boundary is set perpendicular to the incident wave propagation direction. The distance between the second inclusion and the free boundary is $5 a$ as shown in the figure. The distance between two inclusions, $d$, is taken as $3 a, 5 a$ and $10 a$, same as in the infinite matrix problem. Numerical results are shown in Figs. 10 (a), (b), and (c) for the cases of 


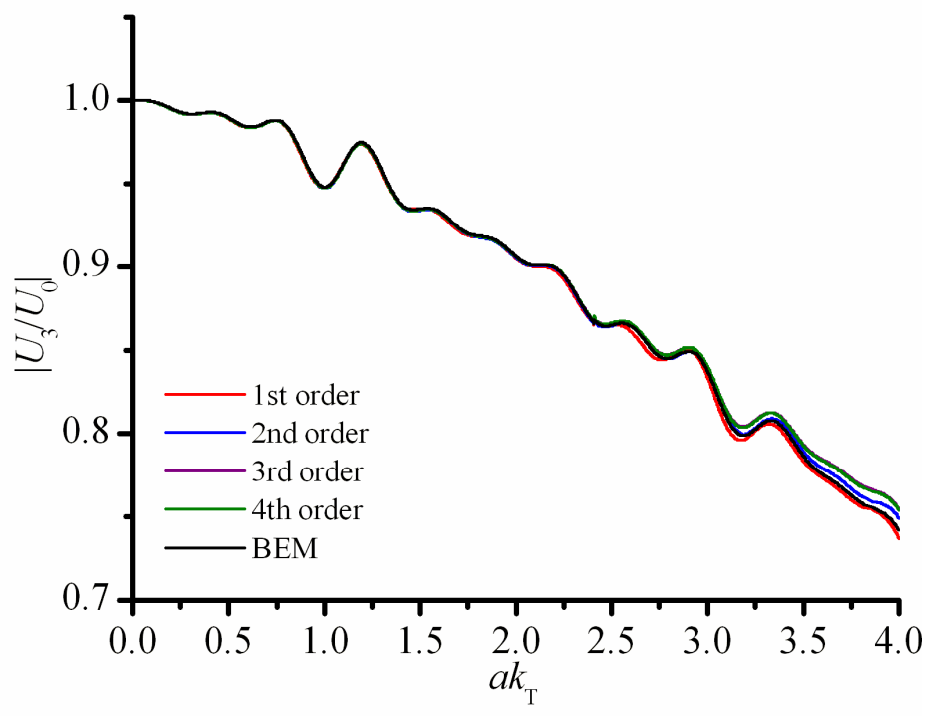

(a) $d=3 a$

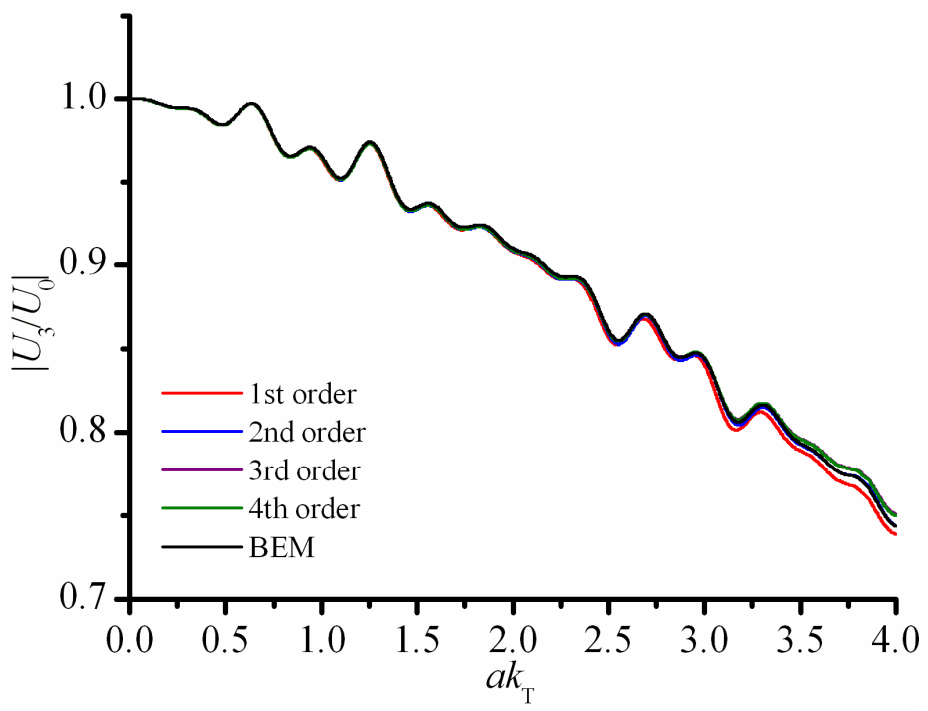

(b) $d=5 a$

Fig. 10 Numerical results of scattered displacement for an anti-plane wave in a half space domain for two inclusions aligned parallel to an incident wave propagation direction, $c_{\mathrm{I}} / c_{\mathrm{M}}=1.05$. 


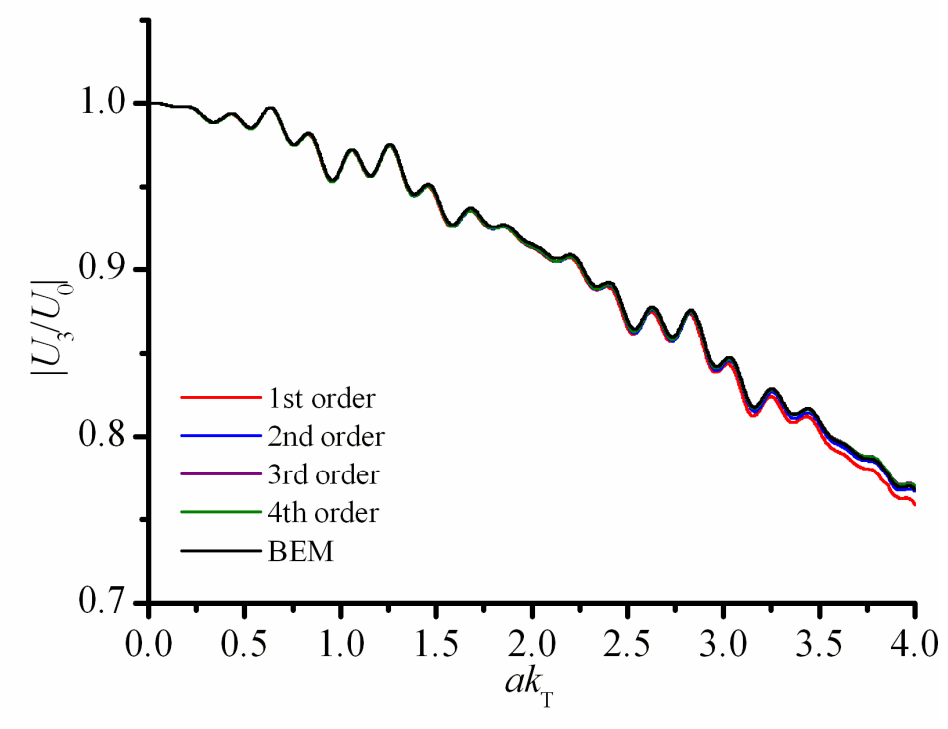

(c) $d=10 a$

Fig. 10 (continue) Numerical results of scattered displacement for an anti-plane wave in a half space domain for two inclusions aligned parallel to an incident wave propagation direction, $c_{\mathrm{I}} / c_{\mathrm{M}}=1.05$.

$d=3 a, 5 a$, and $10 a$, respectively. The reflected wave displacement decreases with increasing frequency. For higher wave number, the wavelength is smaller. Therefore, the incident wave is more scattered due to stronger interaction with inclusions. This scattering process results in a lower reflected wave.

The relative error of the approximation from the BEM is shown in Fig. 11. The error of these results is obviously different for each order approximation. It is obvious that the $3^{\text {rd }}$ and the $4^{\text {th }}$ order approximations show better results than those of the $1^{\text {st }}$ and $2^{\text {nd }}$ order approximations. The error increases with increasing wave number as expected. And the errors decrease with increasing distance, $d$, between two inclusions. 


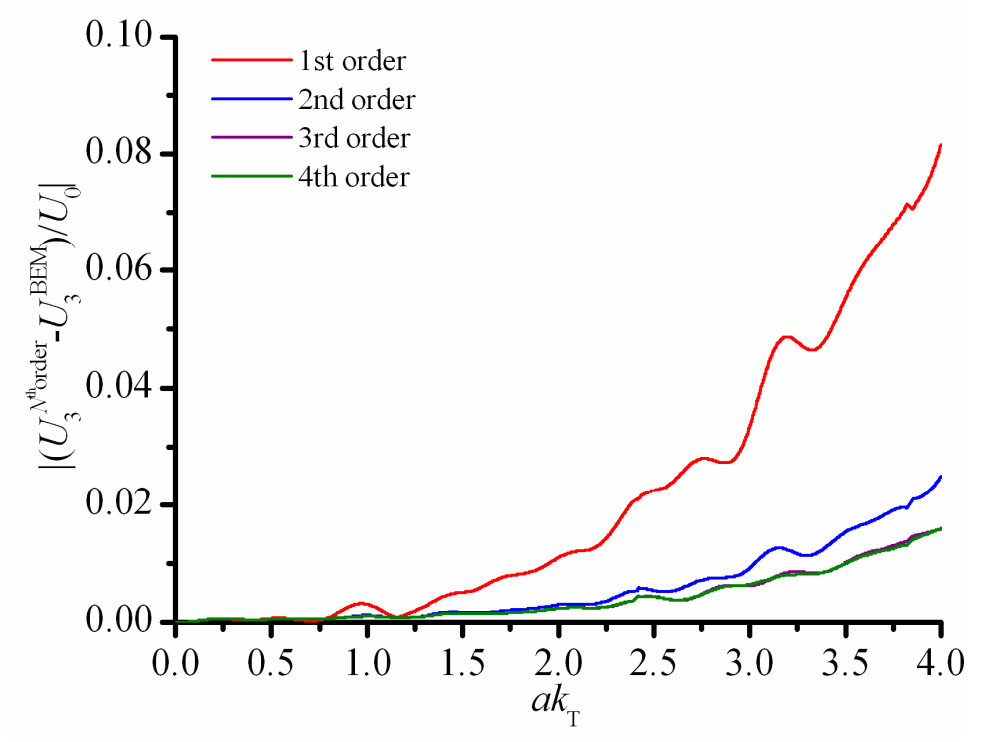

(a) $d=3 a$

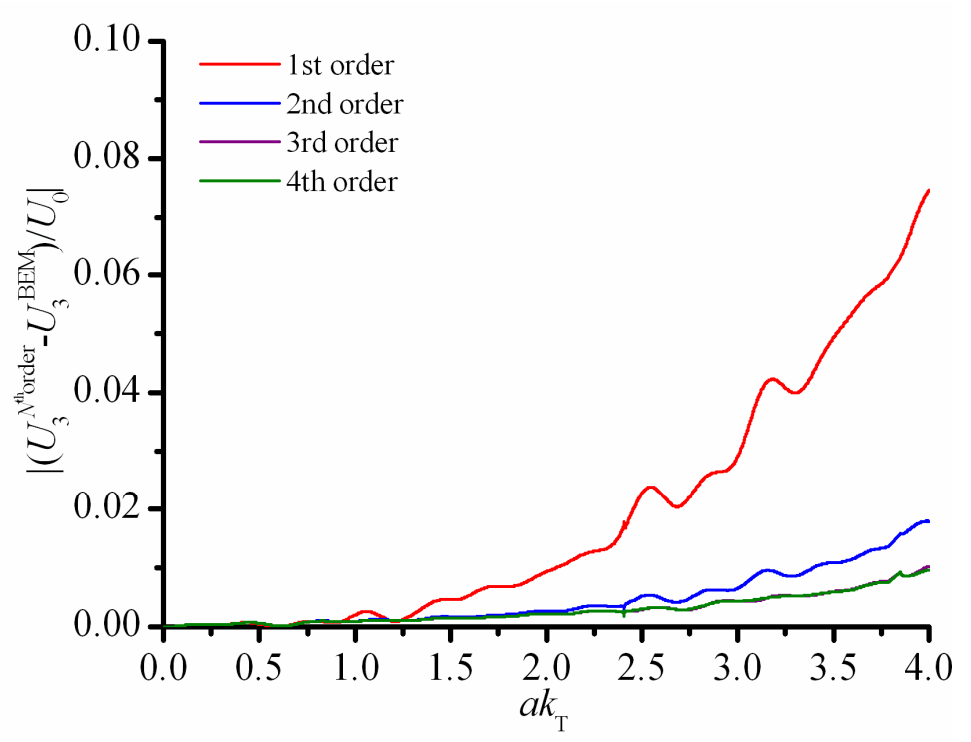

(b) $d=5 a$

Fig. 11 Relative error of the result of scattered displacement for an anti-plane wave in a half space domain for two inclusions aligned parallel to an incident wave propagation direction, $c_{\mathrm{I}} / c_{\mathrm{M}}=1.05$. 


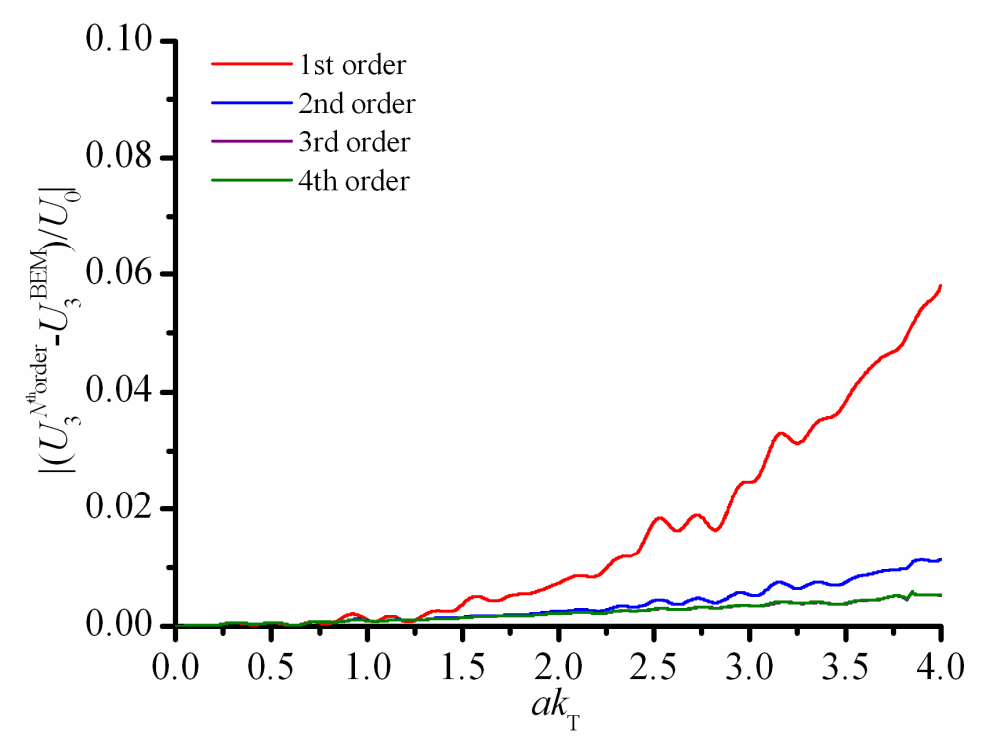

(c) $d=10 a$

Fig. 11 (continue) Relative error of the result of scattered displacement for an anti-plane wave in a half space domain for two inclusions aligned parallel to an incident wave propagation direction, $c_{\mathrm{I}} / c_{\mathrm{M}}=1.05$.

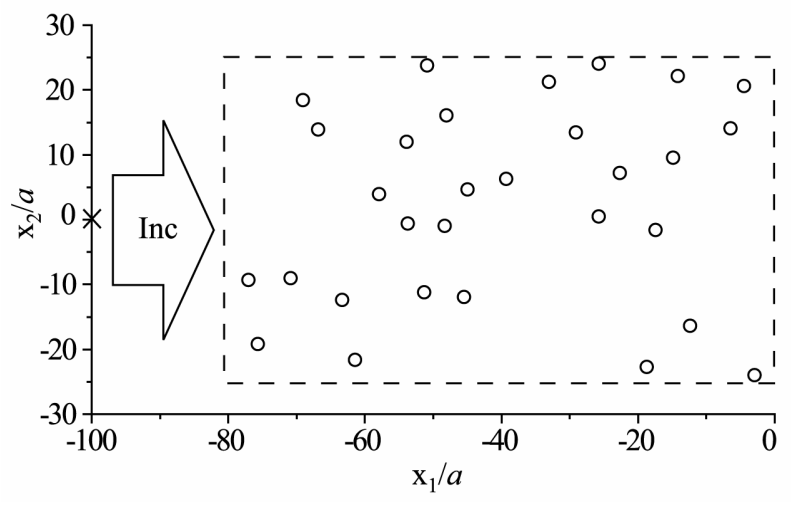

Fig. 12 Thirty inclusions randomly distributed in $80 a \times 50 a$ area in an infinite matrix 


\subsection{Thirty-inclusion model}

The model with 30 inclusions randomly distributed in the area of $80 a \times 50 a$ in an infinite matrix is shown in Fig. 12. The anti-plane wave is incident and the scattered displacement at the specific point $(0,0)$ is calculated. The numerical displacements normalized by incident wave at calculation point of the approximation comparing to those of the BEM are shown in Fig. 13. The error of the approximation is shown in Fig. 14. The relative error in Fig. 14 shows larger error for the $1^{\text {st }}$ order approximation and smaller error for the $3^{\text {rd }}$ and $4^{\text {th }}$ order approximations.

For the half-space, the model of 30 inclusions randomly distributed in an area of $80 a \times 50 a$ is shown in Fig. 15 . The free boundary is again set at $\mathrm{x}_{1}=0$. The numerical displacements calculated at the calculation point normalized by incident wave are shown in Fig. 16. From this figure, it is obvious that the results obtained using $3^{\text {rd }}$ and $4^{\text {th }}$ order approximations show very good agreement with the BEM results. The error of these results, shown in Fig. 17, shows only little difference between the $3^{\text {rd }}$ and $4^{\text {th }}$ order

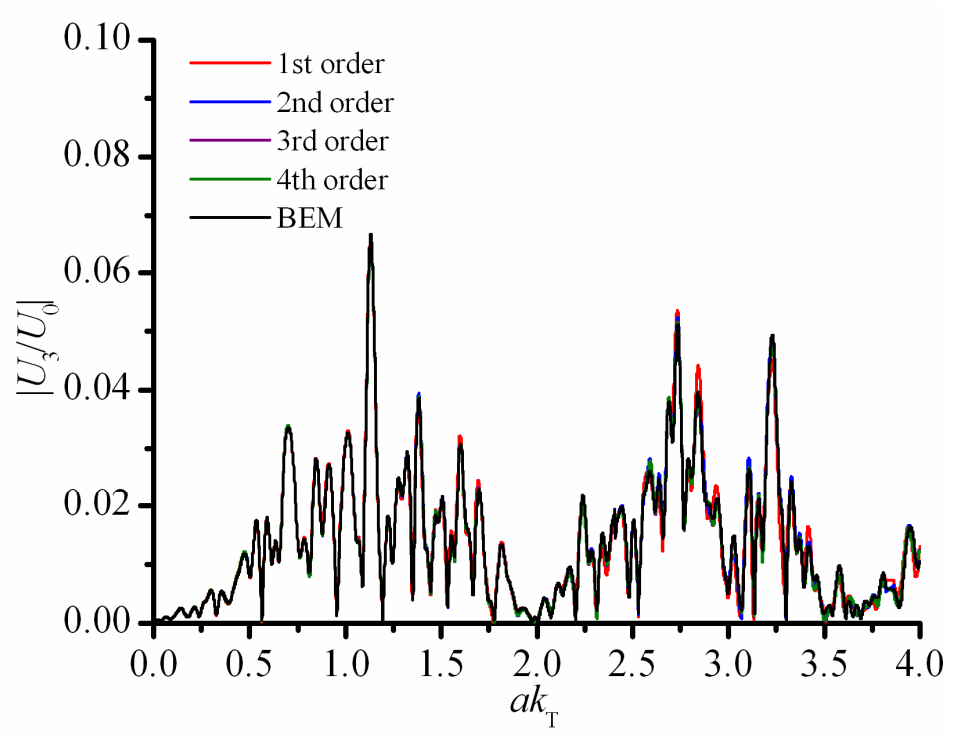

Fig. 13 Numerical results for an anti-plane wave for 30 inclusions randomly distributed in $80 a \times 30 a$ area in an infinite matrix, $c_{\mathrm{I}} / c_{\mathrm{M}}=1.05$. 


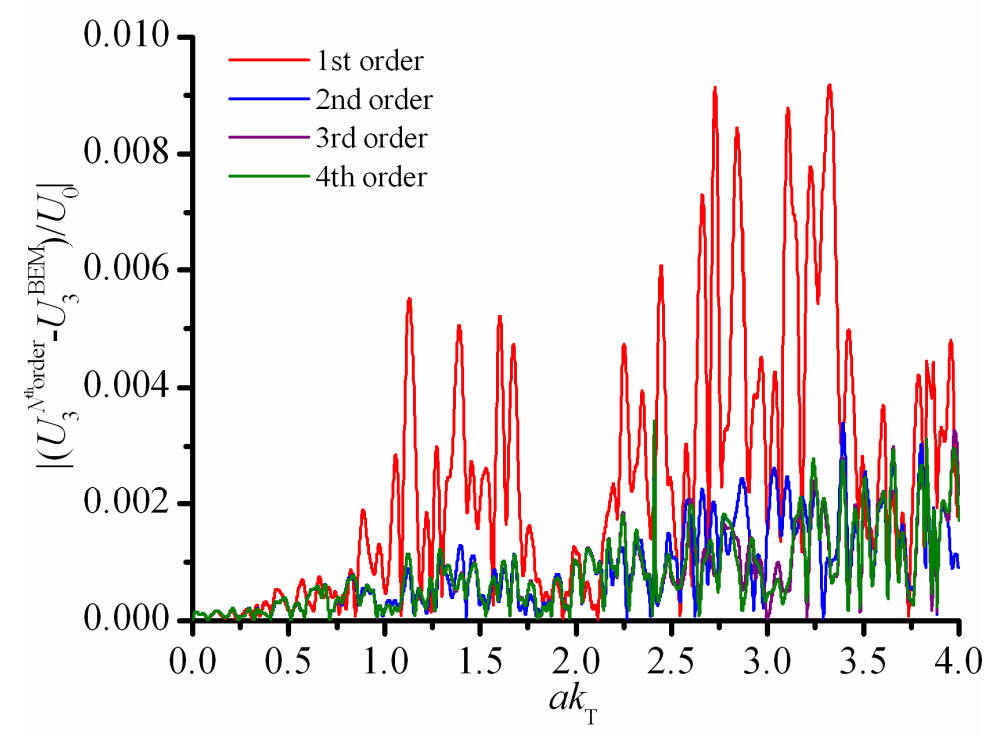

Fig. 14 Relative error of the result of an anti-plane wave for 30 inclusions randomly distributed in $80 a \times 30 a$ area in an infinite matrix, $c_{\mathrm{I}} / c_{\mathrm{M}}=1.05$.

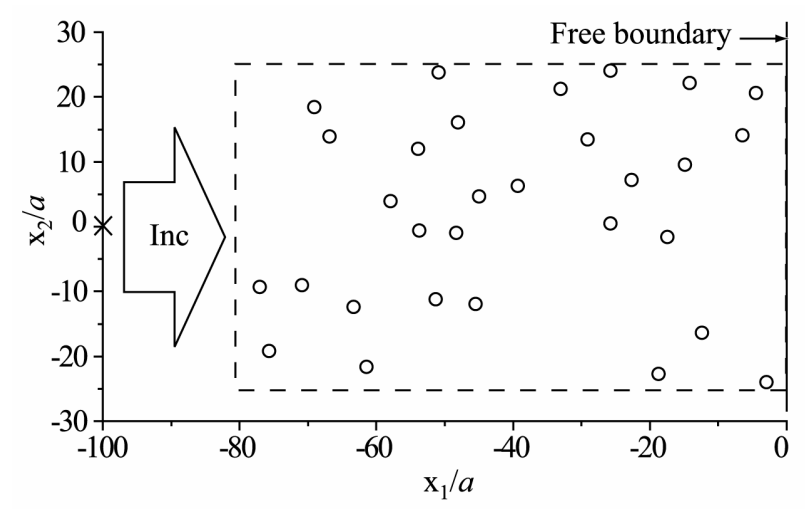

Fig. 15 Thirty inclusions randomly distributed in $80 a \times 50 a$ area in a half-space domain

approximations for wave numbers higher than 3.5. Therefore, to reduce the computation time, latter numerical analyses will be performed up to the $3^{\text {rd }}$ order only. 


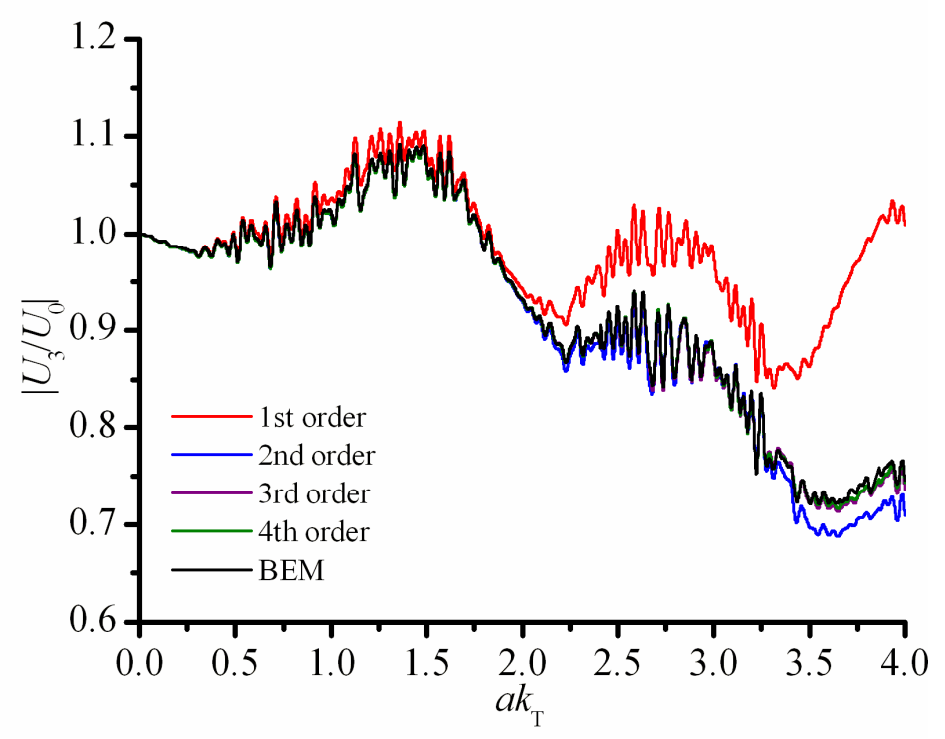

Fig. 16 Numerical results for an anti-plane wave for 30 inclusions randomly distributed in $80 a \times 50 a$ area in a half-space domain, $c_{\mathrm{I}} / c_{\mathrm{M}}=1.05$.

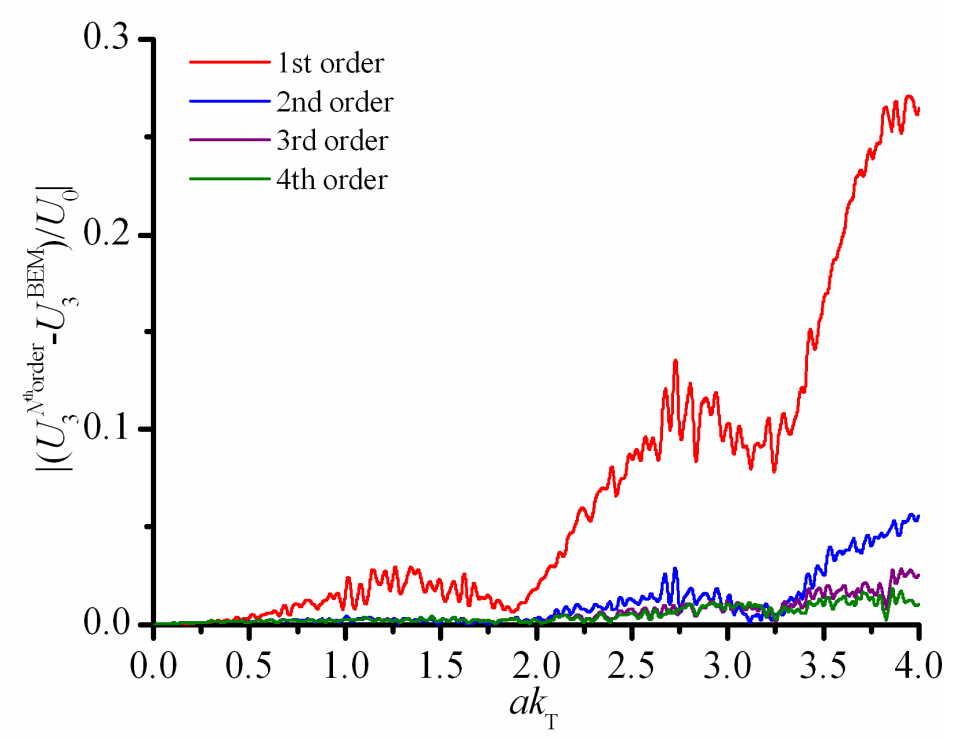

Fig. 17 Relative error of the result of an anti-plane wave for 30 inclusions randomly distributed in $80 a \times 50 a$ area in a half-space domain, $c_{\mathrm{I}} / c_{\mathrm{M}}=1.05$. 


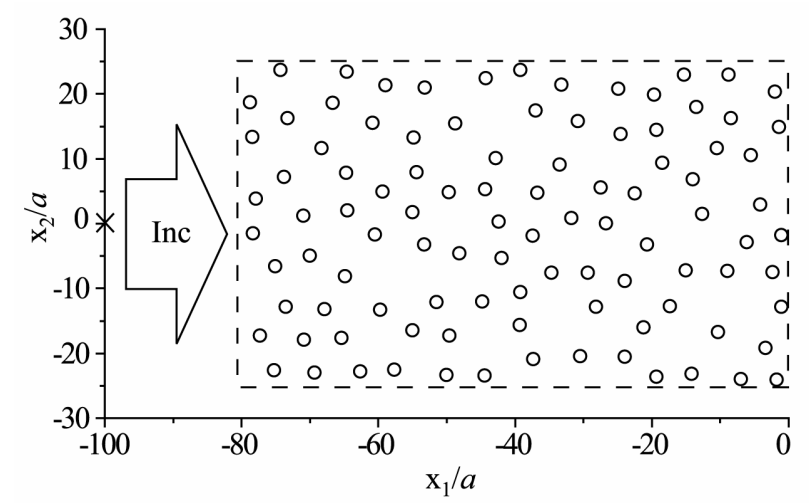

Fig. 18 Hundred inclusions randomly distributed in $80 a \times 50 a$ area in an infinite

matrix

\subsection{Hundred-inclusion model}

The model with 100 inclusions randomly distributed in the area of $80 a \times 50 a$ in an infinite matrix is shown in Fig. 18. The anti-plane wave is incident from the left and the scattered displacement at the specific point $(0,0)$ is calculated. The numerical results calculated up to $3^{\text {rd }}$ order approximation and BEM are shown in Fig. 19. The relative errors are shown in Fig. 20.

In the case of the half-space domain, the numerical model and the results are shown in Fig. 21 and Fig. 22, respectively. The multiple scattered displacements of the $3^{\text {rd }}$ order approximation show good agreement to those of the BEM for $a k$ less than 2.5. The relative errors are shown in Fig. 23.

Assuming the radius of inclusion, $a$, is 50 micron, $c_{\mathrm{M}}=3000 \mathrm{~m} / \mathrm{s}, c_{\mathrm{I}}=3150 \mathrm{~m} / \mathrm{s}$ and the incident wave is one loop of $5 \mathrm{MHz}$ sine wave, the numerical results in the frequency domain can be converted to the time domain results by using inverse Fourier transform technique. The time domain results for the 100 randomly distributed inclusions are shown in Fig. 24 and Fig. 25 for the infinite domain and the half-space domain, respectively. For infinite domain, only the wave scattered directly from inclusions is presented. For half-space domain, the wave scattered directly from inclusions, from free boundary and from inclusions after scattered by free boundary are 


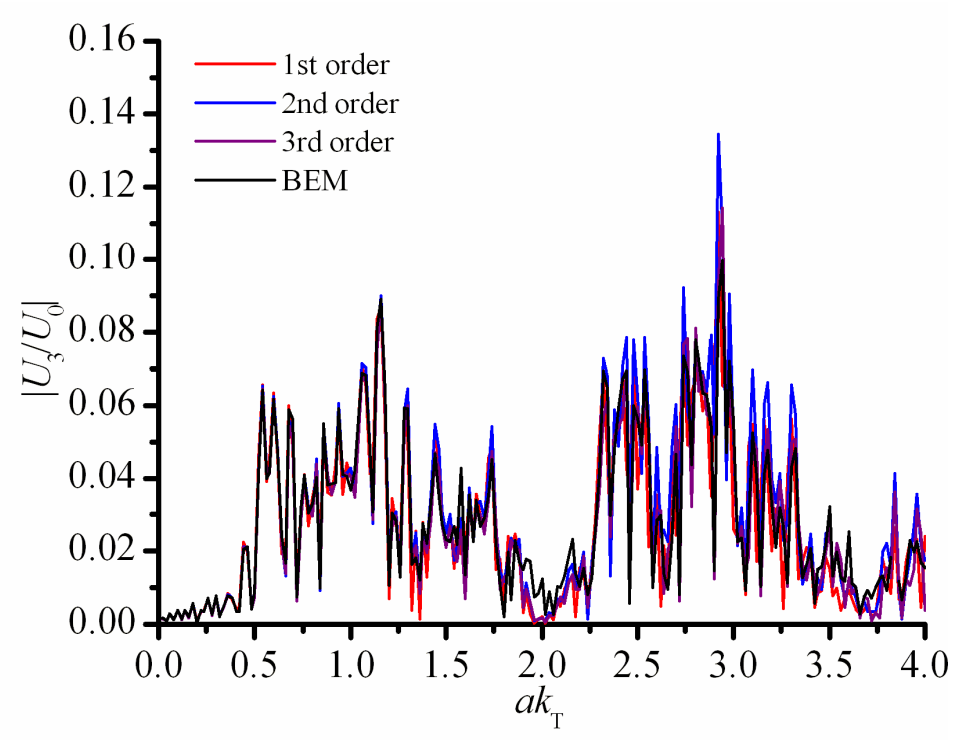

Fig. 19 Numerical results for an anti-plane wave for 100 inclusions randomly distributed in $80 a \times 50 a$ area in an infinite matrix, $c_{\mathrm{I}} / c_{\mathrm{M}}=1.05$.

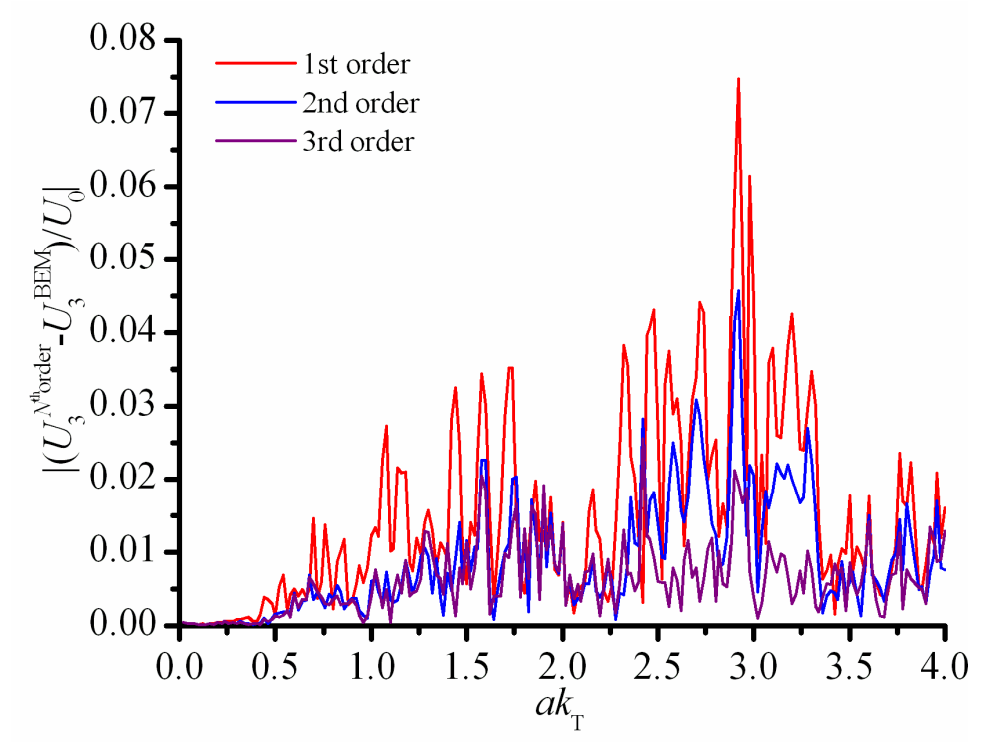

Fig. 20 Relative error of the result of an anti-plane wave for 100 inclusions randomly distributed in $80 a \times 50 a$ area in an infinite domain, $c_{\mathrm{I}} / c_{\mathrm{M}}=1.05$. 


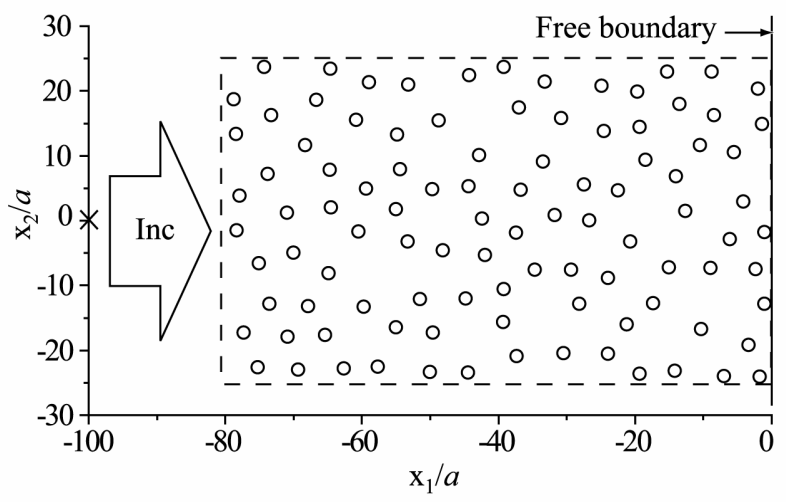

Fig. 21 Hundred inclusions randomly distributed in $80 a \times 50 a$ area in a half-space domain

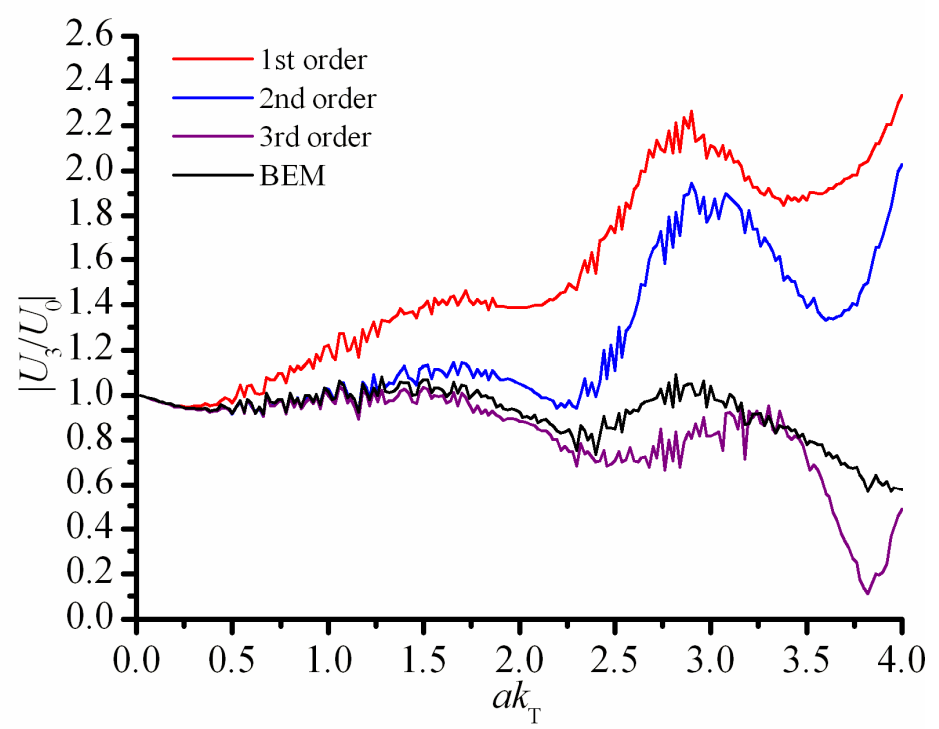

Fig. 22 Numerical results for an anti-plane wave for 100 inclusions randomly distributed in $80 a \times 50 a$ area in a half-space domain, $c_{\mathrm{I}} / c_{\mathrm{M}}=1.05$. 


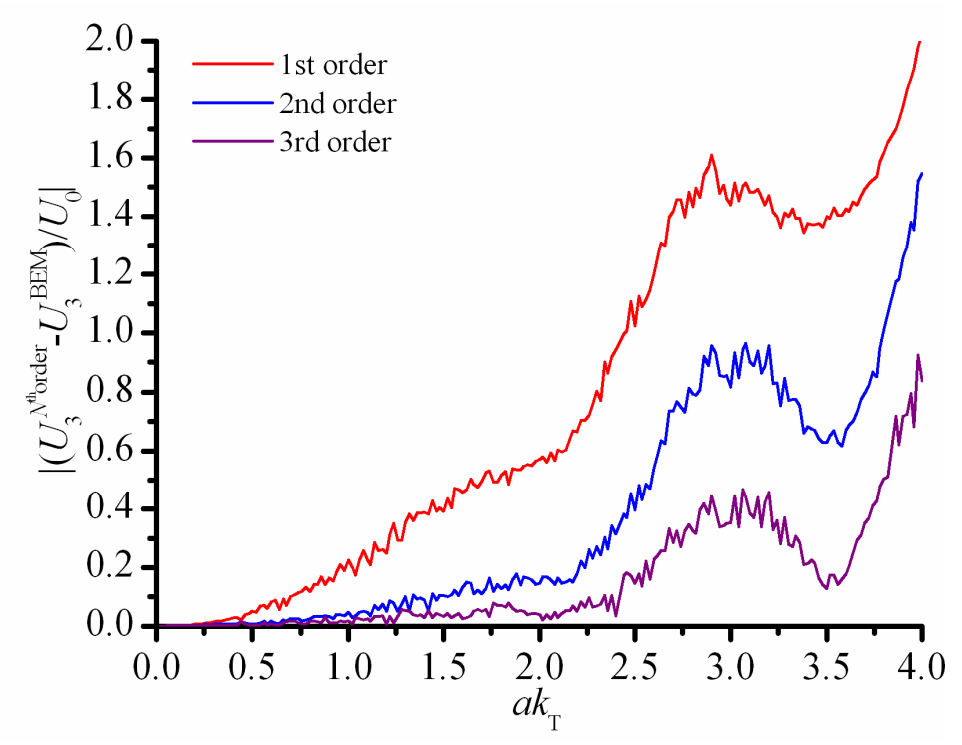

Fig. 23 Relative error of the result of an anti-plane wave for 100 inclusions randomly distributed in $80 a \times 50 a$ area in a half-space domain, $c_{\mathrm{I}} / c_{\mathrm{M}}=1.05$.

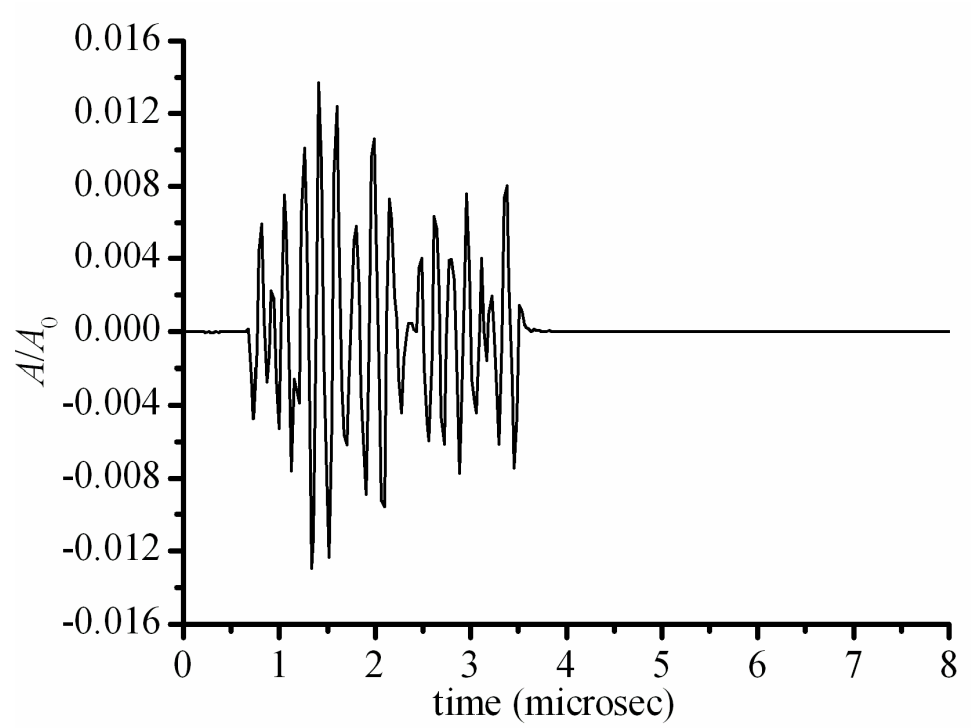

Fig. 24 Time-domain numerical results for an anti-plane wave for 100 inclusions randomly distributed in $80 a \times 50 a$ area in an infinite matrix 

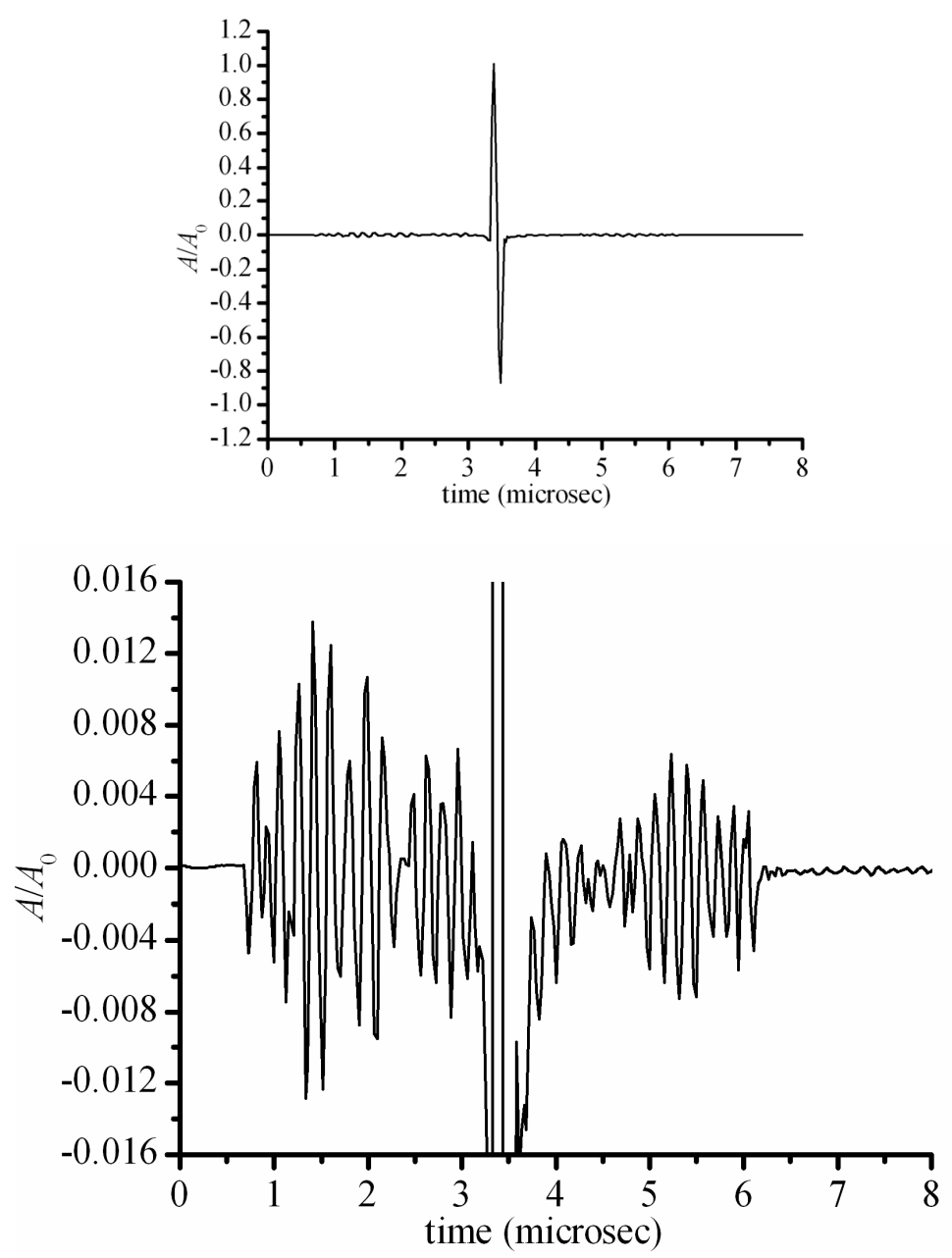

Fig. 25 Time-domain numerical results for an anti-plane wave for 100 inclusions randomly distributed in $80 a \times 50 a$ area in a half-space domain (Top: full-size scale, Bottom: enlarged scale)

presented. Comparing Fig. 24 and Fig. 25, the results from 0 to 3 microseconds are almost the same. This shows that the scattered waves from inclusions at the early stage before the effect of back wall echo are the same for both analyses in the infinite domain and the half-space. 
Table 1 Computational time

\begin{tabular}{|l|c|c|c|}
\hline & $3^{\text {rd }}$ order & $4^{\text {th }}$ order & BEM \\
\hline Infinite domain & & & \\
\hline-2 inclusions & 0.03 & 0.03 & 0.09 \\
\hline-30 inclusions & 0.07 & 1.51 & 96.86 \\
\hline-100 inclusions & 1.74 & 173.36 & 595.24 \\
\hline Half-space domain & & & 0.24 \\
\hline-2 inclusions & 0.03 & 0.03 & 105.98 \\
\hline-30 inclusions & 0.40 & 25.32 & 3489.66 \\
\hline-100 inclusions & 14.02 & 2783.40 & Unit: \\
\hline
\end{tabular}

Unit: second

\subsection{Computational time and memory usage}

Table 1 shows the numerical computational times of the multiple scattering approximation compared to those of the conventional BEM. The number of boundary elements is 40 elements per inclusion. By using multiple scattering approximation the computational time can be reduced.

BEM requires large memory to make the matrix. The size of the matrix depends on the number of elements. For anti-plane wave, the matrix size is $((2 \times n \times m) \times(2 \times n \times m))$ where $\mathrm{n}$ is the number of elements per inclusion and $\mathrm{m}$ is the number of inclusions. The multiple scattering approximation method requires less memory to store the directional parts of the single scattering displacement. The multiple scattering approximation can be used to reduce the computational time and memory usage.

\section{Conclusion}

The multiple scattering approximation of an anti-plane elastic wave is proposed for randomly distributed inclusions in the infinite and the half-space domain. The scattering 
displacement is obtained by summation of the scattering displacement of all possible wave scattering propagation paths. Each wave scattering propagation path is calculated by multiplying the single scattering displacement in sequence. The single scattering displacement is obtained by using the boundary element method with farfield approximation. The numerical results of the multiple scattering approximation of anti-plane wave show a very good agreement to those of BEM.

\section{References}

[1] J. Kraütkramer and H. Kraütkramer, Ultrasonic testing of materials, Springer-Verlag, (1990)

[2] J. Chen, Y. Shi, S. Shi, "Noise analysis of digital ultrasonic nondestructive evaluation system”, Int. J. of Pressure Vessels and Piping 76, 619-630 (1999).

[3] S. Biwa, S. Yamamoto, F. Kobayashi, N. Ohno, "Computational multiple scattering analysis for shear wave propagation in unidirectional composites", Int. J. of Solids and Structures 41, 435-457 (2004).

[4] T. Saitoh, K. Nagai, S. Hirose, "Elastic wave analysis of composite materials using fast multipole boundary element method", Transc. of Jpn. Soc. for Computational Methods in Engineering 5, 37-42 (2005). (in Japanese)

[5] L. W. Cai, J. H. Williams Jr., "Large-scale multiple scattering problems", Ultrasonics 37, 453-462 (1999).

[6] L. W. Cai, J. H. Williams Jr., "Full-scale simulations of elastic wave scattering in fiber-reinforced composites", Ultrasonics 37, 463-482 (1999).

[7] J. E. Gubernatis, E. Domany, "The Born approximation in the theory of the scattering of elastic waves by flaws", J. Appl. Phys. 48, 2812-2819 (1977).

[8] J. H. Rose, J. M. Richardson, "Time domain Born approximation", J. of Nondestructive Evaluation 3 (1), 45-53 (1982).

[9] J. E. Gubernatis, E. Domany, "Long-wave approximations for the scattering of elastic waves from flaws with applications to ellipsoidal voids and inclusions", J. Appl. Phys. 50 (6), 4046-4058 (1979). 
[10] M. Darmon, P. Calmon, B. Bele, "An integrated model to simulate the scattering of ultrasounds by inclusions in steels", Ultrasonics 42, 237-241 (2004).

[11] G. D. Manolis, D. E. Beskos, Boundary element methods in elastrodynamics, Unwin Hyman, (1988).

[12] J. D. Achenbach, A. K. Gautesen, H. McMaken, Ray methods for waves in elastic solids, Pitman, (1982). 